\title{
An Applied Research on Enterprise Internal Performance Appraisal of Knowledge Sharing Using Quantum Neural Networks
}

\author{
Yan Qu \\ Department of MBA \\ Party School of the Liaoning Provincial Committee of CPC \\ Shenyang, China
}

\begin{abstract}
The paper analyzes factors affecting enterprise internal technical knowledge sharing and their relative influence. The research argues that willingness to share knowledge, absorption ability, trust between employees, corporate culture, and recessive features of knowledge have direct impact upon knowledge sharing benefits, which is further affected by the knowledge sharing mechanism. Based on the characteristics of knowledge sharing and influence factors, the paper combines quantum optimization algorithm with neural network. Thus, an evaluation model of knowledge sharing factors is established based on the quantum neural network, and the algorithm is given. Ten enterprises are then selected to test the effectiveness of the model.
\end{abstract}

Keywords-quantum BP neural network; knowledge sharing; effectiveness; factor analysis

\section{INTRODUCTION}

With the rise of knowledge economy around the world, tremendous changes have taken place in business and management environment. Knowledge management has become the core of business management, with knowledge as the primary factor for productivity. As an important part in the knowledge management process, knowledge sharing plays a key role in building competitive advantage for many organizations. Enterprises often encounter obstacles in the implementation of internal knowledge-sharing process. Therefore, it is particularly important to know how to achieve internal knowledge sharing effectively and what are the factors that influence knowledge sharing performance. In recent years, some domestic scholars on knowledge sharing performance evaluation have conducted valuable research and achieved fruitful results. One example is the research of Wang Junxia and Guan Jiancheng, who use the compound DEA method to measure and evaluate enterprise knowledge management effectiveness. The method can pinpoint the gap between the measured enterprise and those in the same industry, track its reasons, and thus offer enterprises an organizational behavior perspective to examine knowledge management activities and related problem and causes.

Quantum neural network is a new concept. Research since the 1990s shows that: information process in the brain may be associated with quantum phenomenon, and quantum mechanics may exist in human brains since it shows similar kinetics features of biological neural networks. This paper aims to show that the combination of artificial neural network and quantum optimization theory of quantum neural network can better simulate the human information processing mechanism. Thus, its application to internal knowledge sharing influence factors evaluation may promote knowledge sharing in enterprise benefit and work as a reference to increasing enterprise innovation capacity.

\section{INFLUENCE FACTORS OF INTERNAL KNOWLEDGE SHARING PERFORMANCE}

Knowledge sharing refers to the transfer of knowledge from one to another, involving two main bodies of knowledge. The provider and receiver of knowledge will determine the knowledge sharing benefits.The sharing of tacit knowledge is mainly dependent on the individual, so the exchanges and learning of internal members, as well as interaction with external environment should be the focus of knowledge sharing. Many factors may affect internal knowledge sharing the benefits, including lack of trust, culture and language differences, time limitation, the difficulty of enterprise knowledge sharing, incentive mechanism, the staff's imparting and absorptive capacity. The purpose of the paper is to analyze the impact of internal factors on knowledge sharing within enterprise. The paper argues that knowledge sharing within enterprise benefit is mainly affected by staff's desire of knowledge sharing, employee's knowledge absorptive capacity, employee trust relations, corporate culture, and the tacitness of knowledge sharing.

\section{A. Employee's Desire for Knowledge Sharing}

Knowledge employee is the source of sharing desire and knowledge transfer; his willingness to share will determine the quality and effectiveness of knowledge sharing. In an enterprise, a unique knowledge means power, so knowledge owners worry that sharing knowledge will lose the unique value of the knowledge. As a result, they may not be willing to take the initiative to share knowledge. Employee's knowledge sharing willingness not only determines whether the knowledge provider is able to provide qualified valuable 
knowledge, but also affects the knowledge receiver's knowledge acquisition and absorption as well. The desire for knowledge sharing is a key determinant in successful knowledge sharing. Most tacit knowledge is carried by people, and strong knowledge sharing desire and ability contribute to the knowledge sharing benefit.

\section{B. Employee's Capacity of Knowledge Absorbing}

The success of knowledge sharing depends on not only the provider, but also the receiver's absorptive capacity. Knowledge absorptive capability can make the receiver achieve successful absorption, digestion and application of new knowledge. In the process of cooperation, absorptive capacity is the implementation of knowledge sharing. If knowledge is only transferred, enterprises may only use the knowledge without absorbing, storing and diffusing, which will lead to negative effects on learning performance. Related studies indicate that both personal and organizational absorptive capacity has positive impact on knowledge sharing effectiveness.

\section{Trust between the Employees}

It is difficult to ensure benefits of cooperation when internal trust is not constructed. Trust is a person's willingness to take risks for a specific subject, as well as a way to gain trust from others. To enterprise workers with internal knowledge, knowledge sharing may result in the loss of one's own competitive advantage, and it may even pose a threat to oneself. Therefore, knowledge sharing behavior will only take place when the knowledge owner is willing to take the risk, and this willingness shows trust in others. If all internal staff is willing to put collective benefit before personal interests and openly share knowledge and information based on mutual trust, the enterprise internal knowledge sharing benefit will get promoted considerably.

\section{Corporate Culture}

The degree of trust among employees will have an impact on their work attitude and technology learning. Corporate culture is the code of conduct and evaluation criterion for the enterprise and its staff, which guides employee behavior. A trusting corporate culture can directly or indirectly affect the knowledge sharing behavior among employees. The key to a facilitative environment of knowledge sharing lies in the establishment of a corporate culture of knowledge sharing.

\section{E. Sharing of Tacit Knowledge}

Knowledge production in the most prominent characteristic is the human brain. Explicit knowledge is just the tip of the iceberg, and a large amount of knowledge is below the surface. In fact, tacit knowledge exists not only in individuals, but also exists in team work, special specifications, knowledge circulation, decision-making process, corporate culture and organization communication system. Tacit knowledge is hard to pass on to other individuals and organizations through high text and special symbols; it must be learned through special methods over a long time. Therefore, tacit knowledge intensity will affect knowledge sharing benefits.

\section{Quantum Neural NeTwork Model}

In 1995, Prof. Kak in Louisiana State University, U.S., first put forward the concept of quantum neural computation. He pointed out a new computing paradigm combining neural computing and quantum computing, and thus became the founder of the theory. Prof. Kak suggested that this people may be of great help to the study of human consciousness. In 1998, T.Menner's research demonstrated that quantum neural network has better performance than the traditional neural network.

It remains a controversial issue as to whether quantum neural network is superior to classical neural network or not, but some studies have shown that quantum neural network at least has the same computational ability as the classical neural network. There have been some research results showing that the quantum neural networks in many ways are superior to classical neural network. Through the joint efforts of researchers around the world, research on quantum computation and neural network has changed from skepticism to optimism, from simple quantum artificial neural network to the relatively complex brain functions in quantum theory, also from pure theoretical stage to a simple experiment application stage. A variety of quantum neural network models also appeared subsequently.

Based on features of knowledge sharing evaluation, this paper chooses a quantum BP neural network model, and constructs a three-layer quantum neural network (Figure1). Each input, hidden and output layer has n, p, m quantum neurons.

Layer One: input layer, His every node represents one input variables (Factors influencing the performance), $\left|x_{n}\right\rangle$ is the network input.

Layer Two: hidden layer, $\mathrm{R}(\theta \mathrm{ij})$ and $\mathrm{T}(\omega \mathrm{jk})$ are expressed as regulating the input, hidden layer quantum phase gate. $\mathrm{C}(0) 、 \mathrm{U}(\alpha \mathrm{j})$ and $\mathrm{U}(\beta \mathrm{k})$ is the input layer, hidden layer and output layer incentive function of the controlled not gate. $\left|h_{i}\right\rangle$ is the hidden layer output.

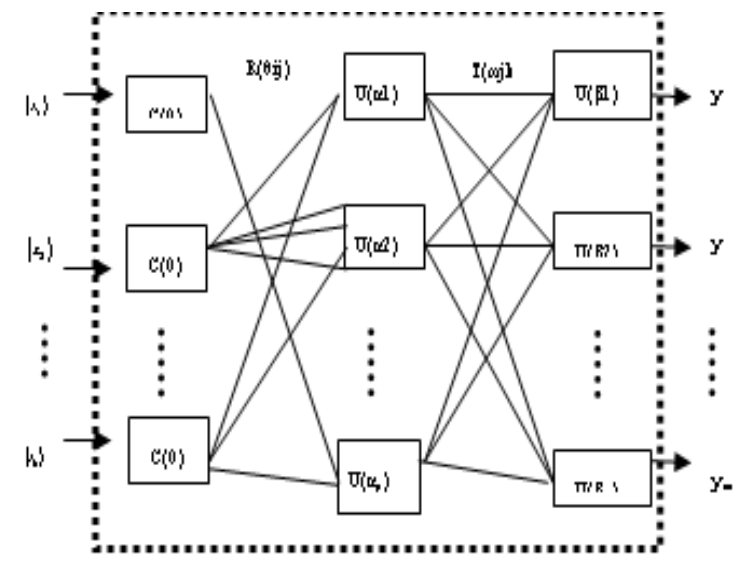

Fig. 1. Three layer quantum BP neural network mode. 
The relationship between input and output is as follows:

$$
\begin{gathered}
\left|h_{j}\right\rangle=U\left(\alpha_{j}\right)\left(\sum_{i=1}^{n} R\left(\theta_{i j}\right)\left|x_{i}\right\rangle\right) \\
\left|y_{k}\right\rangle=U\left(\beta_{k}\right)\left(\sum_{j=1}^{p} T\left(\omega_{i j}\right)\left|h_{i}\right\rangle\right)=U\left(\beta_{k}\right)\left(\sum_{j=1}^{p}\left(T\left(\omega_{j k}\right)\right)\left(U\left(\alpha_{j}\right)\left(\sum_{i=1}^{n} R\left(\theta_{i j}\right)\left|x_{i}\right\rangle\right)\right)\right) \\
\mathrm{i}=1, \ldots \ldots, \quad \mathrm{n} ; \mathrm{j}=1, \quad \ldots \ldots, \quad \mathrm{p} ; \mathrm{k}=1, \ldots \ldots, \quad \mathrm{m} .
\end{gathered}
$$

The quantum description of training samples:

In order to achieve the training sample quantum state description, on n-dimensional Euclidean space to real-valued vector describing the training samples,

$\bar{X}=\left(\overline{x_{1}}, \overline{x_{2}}, \ldots, \overline{x_{n}}\right)$. The conversion formula is defined as follows:

$$
|X\rangle\left[\left|x_{1}\right\rangle,\left|x_{2}\right\rangle, \ldots,\left|x_{n}\right\rangle\right]^{T}
$$

Including

$$
\left|x_{i}\right\rangle=\cos \left(\frac{2 \pi}{1+\exp \left(-\overline{x_{i}}\right)}\right)|0\rangle+\sin \left(\frac{2 \pi}{1+\exp \left(-\overline{x_{i}}\right)}\right)|1\rangle=\left[\cos \left(\frac{2 \pi}{1+\exp \left(-\overline{x_{i}}\right)}\right) \sin \left(\frac{2 \pi}{1+\exp \left(-\bar{x}_{i}\right)}\right)\right]^{T}
$$

Error function:

$$
E=\frac{1}{2}(\tilde{Y}-Y)^{2}
$$

Parameter adjustment rules:

$$
\begin{gathered}
\beta_{k}(t+1)=\beta_{k}(t)+\eta \Delta \beta_{k}(t) \\
\omega_{j k}(t+1)=\omega_{j k}(t)+\eta \Delta \omega_{j k}(t) \\
\alpha_{j}(t+1)=\alpha_{j}(t)+\eta \Delta \alpha_{j}(t) \\
\theta_{i j}(t+1)=\theta_{i j}(t)+\eta \Delta \theta_{i j}(t)
\end{gathered}
$$

The $\eta$ is the learning rate.

The algorithm is as follows:

- The input samples for quantum state description.

- Network parameter initialization: each layer unit number, phase parameter $\theta_{\mathrm{ij}}$ and $\omega_{\mathrm{jk}}$, turning parameter $\alpha_{j}$ and $\beta_{k}$, learning rate $\eta$, limit error $\varepsilon$, limit the number of iteration $\mathrm{N}$; the current iteration step number $\mathrm{t}=0$.

- According to (1), (2) two type calculation of each layer output; according to (5) - (8) type correction network parameters.

- According to (4) the calculation error of E type, If $\mathrm{E}<\varepsilon$ or $\mathrm{t}>\mathrm{N}$, network training ends, or $\mathrm{t}=\mathrm{t}+1$ turns on one step to continue training.

- Save parameters: $\theta_{\mathrm{ij}}, \omega_{\mathrm{jk}}, \alpha_{\mathrm{j}}, \beta_{\mathrm{k}}$.

\section{EMPIRICAL ANALYSIS}

Based on the above theory and method, a BP enterprise knowledge sharing evaluation model is established based on quantum neural network, with enterprise knowledge sharing benefit influence factors (employee sharing, absorptive capacity, trust relationship, culture and knowledge implicit) as input variables.

Data are collected from a questionnaire survey. Sample selection primarily targets at firms who have an independent R\&D or technical department, or those specializing in new product development. 1000 questionnaires are collected from a total of 10 enterprises with effective recovery of 735 . We take data from 8 enterprises as training samples to train a neural network, and use data from the other 2 companies as test samples to examine the result of the model. By using BP neural network modeling, a maximum number of iterations of $\mathrm{N}=1000$, the population size of $\mathrm{T}=10, \mathrm{P}=0.5$ mutation probability, rotation angle $\theta=0.16$, the set of parameters $\eta \in\left(\begin{array}{ll}0.5 & 0.35\end{array}\right), \alpha \in(0.70 .6), \mathrm{E}<0.001$. After calculation and comparative analysis, the quantum BP neural network finally gives 5-15-1 optimal network model. Network output results are shown in "Table I".

"Magnetization (A (m(1)," not just "A/m." Do not label axes with a ratio of quantities and units. For example, write "Temperature (K)," not "Temperature/K."

TABLE I. NETWORK OUTPUT RESUlTS

\begin{tabular}{|l|l|l|l|l|l|}
\hline & $\mathrm{X} 1$ & $\mathrm{X} 2$ & $\mathrm{X} 3$ & $\mathrm{X} 4$ & $\mathrm{X} 5$ \\
\hline $\begin{array}{l}\text { Output } \\
\text { results }\end{array}$ & 0.65 & 0.516 & 0171 & 0.81 & 0.75 \\
\hline & $\mathrm{X} 6$ & $\mathrm{X} 7$ & $\mathrm{X} 8$ & $\mathrm{~T} 1$ & $\mathrm{~T} 2$ \\
\hline $\begin{array}{l}\text { Output } \\
\text { results }\end{array}$ & 0.054 & 0.329 & 0.242 & 0.57 & 0.272 \\
\hline
\end{tabular}

\section{CONCLUSION}

Knowledge has become the most valuable strategic resource and core element of production. In essence, the heterogeneity of enterprise knowledge and ability is the key competitive advantage. In order to achieve reasonable configuration of enterprise interior knowledge resource, large amounts of scattered knowledge in various internal individuals and groups need to be developed and utilized, so that the acceleration of knowledge sharing can be achieved. Research on enterprise knowledge sharing is of vital importance for enterprises to promote knowledge management. Scientific assessment of various factors impact on team knowledge sharing will help enterprises to understand their knowledge management ability, establish and perfect management, and thus enhance their competitiveness.

Based on previous research results, the paper summarizes and analyzes factors affecting knowledge sharing directly, including willingness to share knowledge, absorption ability, and trust between employees, corporate culture, and recessive features of knowledge. It shows that knowledge sharing benefit is also affected by knowledge sharing mechanism. Combining quantum optimization algorithm with neural network, the paper constructs a quantum neural network, Quantum evolutionary algorithm can reduce the searching complexity, and has the advantage of fast 
convergence speed and strong optimization capability. The paper applies neural network algorithm, which combines quantum computation and quantum neural computation, to the modeling of influential factors of knowledge sharing. Experimental results show that the quantum neural network has faster convergence speed and higher model accuracy.

This research is still at its initial stage, and there are many limitations, such as small sample size, limited number of selected industries, not comprehensive coverage of influence factors, and no discussion about incentive mechanism. More effort will be made in the above respects in future research.

\section{REFERENCES}

[1] Szulanski, G. The process of knowledge transfer: a diachronic analysis of stickiness $[\mathrm{J}]$, Organizational Behavior and Human Decision Process, 2000

[2] Nonaka, I. \& Teece, D. Managing industrial knowledge: ceation, transfer and utilization [M], Hardback: Sage Publication, 2001.

[3] Kak S C. On quantum neural computing, Information Sciences, 1995

[4] Sanjay Gupta, R. K.P.Zia. Quantum neural networks. ArXiv: quant$\mathrm{ph} / 0201144 \mathrm{v} 1,30$ Jan 2002.

[5] LiPanchi, Zhou Hongyan, Modeland Algorithm of Quantum Neural Network Based on the Controlled Hadeamard Gates [J], Journal of Computer Research and Development. 2015(52):211-220

[6] ZHANG J A, EDGAR F, GEARE A, et al. The interactive effects of entrepreneurial orientation andcapability based HRM on firm performance: themediating role of innovation ambidexterity[J]. Indus -trial marketing management, 2016, 59(11):131-143..

[7] LI J J, POPPO L, ZHOU K Z. Relational mecha-nisms, formal contracts and local knowledge acquisition by international subsidiaries[J].Strategic management journal, 2010, 31(4):349-370

[8] YESIL S, KOSKA A, BUYUKBESE T. Knowledge sharing process, innovation capability and innovation performance: an empirical study[J]. Procedia-social and behavioral sciences, 2013, 75(3):217225.

[9] ENKEL E, HEIL S, HENGSTLER M, WIRTH H. Exploratory and exploitative innovation: to what extent do the dimensions of individual level absorptive capacity contribute [J]. Technovation, 2017,60- 61:29-38.

[10] HAGEDOORN J, CLOODT M. Measuring innovative performance: Is there an advantage in using multiple indicators? [J]. Research policy, 2003,32(8):1365-1379.

[11] ACS Z J, AUDRETSCH D B, LEMHMANN E E. The knowledge spillover theory of entrepreneurship[J]. Small business economics, 2013, 41(4):757-774

[12] VARGA A, SCHALK H J. Knowledge spillovers, agglomeration and macroeconomic growth: an empirical approach[J]. Regional studies, 2004, 38(8):977-989

[13] ISAKSSON O H D, SIMETH M, SEIFERT R W. Knowledge spillovers in the supply chain: evidence from the high tech sectors[J].Research policy, 2016, 45(3):699-706.

[14] AZUBUIKE V M U. Technological innovation capability and firm's performance in new product development[J]. Communications of the lima, 2013, 13(1):43-55.

[15] ROSENZWEIG S. The effects of diversified technology and country knowledge on the impact of technological Innovation[J]. Journal of technology transfer,2016(8):1-21.

[16] ANG H, STEENSMA H K. When do firms rely on their knowledge spillover recipients for guidance in exploring unfamiliar knowledge? [J]. Research policy, 2014, 43(9):1496-1507.
[17] AGARWAL R, AUDRETSCH D, SARKAR M. Knowledge spillovers and strategic entrepreneurship[J]. Strategic entrepreneurship journal, 2010, 4(4):271-283.

[18] SONG G, MIN S, LEE S, SEO Y. The effects of network reliance on opportunity recognition: a moderated mediation model of knowledge acquisition and entrepreneurial orientation $[\mathrm{J}]$. Technological forecasting \& social change, 2017,117:98-107.

[19] SANCHEZ M A, ORTIZ-DE-URBINA-CRIADO M, MORAVALENTIN M E. Effects of knowledge spillovers on innovation and collaboration in science and technology parks[J]. Journal of knowledge management, 2011,15(6):948-970

[20] GRILLITSCH M, NILSSON M. Firm performance in the periphery: on the relation between firm-internal knowledge and local knowledge spillovers[J].Regional studies , 2015,40(10):1-13

[21] YASAR M, PAUL C J M J. Firm performance and knowledge spillovers from academic, industrial and foreign linkages: the case of China[J]. Journal of productivity analysis, 2012,38(3):237-253.

[22] CHEN L, XU Q. The mechanism of innovation capability leveraging via strategy in SMEs[C]. Industrial engineering \& engineering management, IEEM 2009. HongKong, 2009:1057-1061.

[23] Kaks. On quantum neural computing[J]. Information Science, 1995,83(3):143-160.

[24] Gopathy P, Niocolaos B. Quantum neural networks(QNNs) inherently fuzzy feedforward neural networks[J]. IEEE Trans on Neural Networks. 1997,8(3):679-693.

[25] Giuliano B, Giulio C, Guiliano S. Principles of Quantum Computation and Information, Volume I:Basic Concepts[M]: Beijing, Science Press,2011:151-178. 